\title{
Influence of Nuclei Segmentation on Breast Cancer Malignancy Classification
}

\author{
Łukasz Jeleń, Thomas Fevens and Adam Krzyżak \\ Department of Computer Science and Software Engineering, \\ Concordia University \\ 1455 de Maisonneuve Blvd. West \\ Montreal, QC H3G 1M8 \\ Canada
}

\begin{abstract}
Breast Cancer is one of the most deadly cancers affecting middle-aged women. Accurate diagnosis and prognosis are crucial to reduce the high death rate. Nowadays there are numerous diagnostic tools for breast cancer diagnosis. In this paper we discuss a role of nuclear segmentation from fine needle aspiration biopsy (FNA) slides and its influence on malignancy classification. Classification of malignancy plays a very important role during the diagnosis process of breast cancer. Out of all cancer diagnostic tools, FNA slides provide the most valuable information about the cancer malignancy grade which helps to choose an appropriate treatment. This process involves assessing numerous nuclear features and therefore precise segmentation of nuclei is very important.

In this work we compare three powerful segmentation approaches and test their impact on the classification of breast cancer malignancy. The studied approaches involve level set segmentation, fuzzy c-means segmentation and textural segmentation based on co-occurrence matrix.

Segmented nuclei were used to extract nuclear features for malignancy classification. For classification purposes four different classifiers were trained and tested with previously extracted features. The compared classifiers are Multilayer Perceptron (MLP), Self-Organizing Maps (SOM), Principal Component-based Neural Network (PCA) and Support Vector Machines (SVM). The presented results show that level set segmentation yields the best results over the three compared approaches and leads to a good feature extraction with a lowest average error rate of $6.51 \%$ over four different classifiers. The best performance was recorded for multilayer perceptron with an error rate of $3.07 \%$ using fuzzy c-means segmentation.
\end{abstract}

Keywords: malignancy grading, nuclear segmentation, Bloom-Richardson scale, breast cancer malignancy classification

\section{INTRODUCTION}

Malignancy grading allows doctors to precisely estimate cancer behavior with or without undertaking treatment and therefore is called a prognostic factor. It plays an important role in breast cancer diagnosis and the appropriate treatment is chosen accordingly to this factor. This is a complicated process that involves assessing numerous nuclear features that allow for malignancy grading. Cancer malignancy grading is frequently based on a numeric scale that was introduced by Bloom and Richardson in 1957. ${ }^{1}$ The grading scheme proposed by the authors was derived to assess malignancy from histological slides and is now widely used by pathologists to grade not only histological but also cytological tissue. According to the Bloom and Richardson scheme, malignancy is evaluated based on three factors. These factors take into consideration different features of the nucleus. Each of the three factors is evaluated on a three-point scale and based on that evaluation, cancer is assigned one of the three grades:

Further author information: (Send correspondence to Łukasz Jeleń)

Łukasz Jeleń: E-mail: 1_jelen@cs.concordia.ca

Thomas Fevens: E-mail: fevens@cs.concordia.ca

Adam Krzyżak: E-mail: krzyzak@cs.concordia.ca

Medical Imaging 2009: Computer-Aided Diagnosis, edited by Nico Karssemeijer, Maryellen L. Giger Proc. of SPIE Vol. 7260, 726014 - (c) 2009 SPIE · CCC code: 1605-7422/09/\$18 · doi: 10.1117/12.811733 
- Grade I - Low malignancy

- Grade II - Intermediate malignancy

- Grade III - High malignancy

These grades are determined as a summation of the awarded points according to Fig. 1 (taken from Bloom and Richardson $\left.{ }^{1}\right)$ :

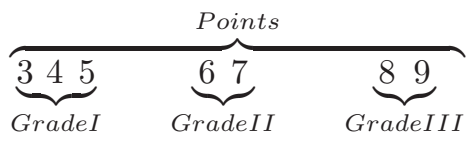

Figure 1. Grade distribution.

When cancer is assigned with one of the three grades, the appropriate treatment is undertaken which assumes precise estimation of malignancy. Malignancy estimation is a subjective procedure which lacks repeatability. ${ }^{2}$ To overcome this problem Bloom-Richardson scheme can be converted into a classification problem, where each class is represented by the cancer malignancy.

In the literature one can find various approaches for breast cancer classification. ${ }^{3-7}$ Most of these approaches involve classification of breast cancer to two classes: benign or malignant. Schnorrenberg et al. ${ }^{6}$ presented an automated approach to classification of breast cancer from histological slides using receptive fields for nuclear segmentation. Their algorithm was able to classify these types of images with $28 \%$ error. Cheng et al. 5,8 presented a parallel approach for grading histological tissue during breast cancer diagnosis. Both of these approaches are attempts of grading and classifying of histological images. In our work we are concerned with cytological examination of a breast which is performed before a histological examination. Approaches presented by Street et al., ${ }^{3,9}$ Walker et al. ${ }^{4}$ and Nezafat et al. ${ }^{10}$ deal with classification of cytological tissue. To date only Street et al. ${ }^{3}$ described an attempt of breast cancer malignancy grading. They also presented a set of nuclear features that are widely used for classification purposes. Attempts by Nezafat et al. involved testing of different classifiers on the database provided by Street et al. ${ }^{9}$ and describing which features are the most discriminatory. Jeleń et al. ${ }^{7}$ presented a system for grading of breast cancer malignancy based on level set segmentation of nuclei from fine needle aspiration biopsies. Level sets have proved their good performance in medical image segmentation. ${ }^{11,12}$

In this work we compare three powerful segmentation approaches and test their influence on the classification of breast cancer malignancy. The compared approaches involve level set segmentation, fuzzy c-means segmentation and textural segmentation based on co-occurrence matrix. All of the segmentation techniques have been shown to be effective for segmentation of medical images. ${ }^{13-17}$ This observation provides motivation for testing their segmentation quality for breast cancer nuclei extraction. Results of the comparison can be found in section 3 followed by conclusions on the influence of these techniques on malignancy classification results. More detailed discussion of the images and methods used in this study is presented in section 2 .

\section{IMAGE SEGMENTATION AND CLASSIFICATION}

Classification is a task of assigning an item to a certain category, called a class, based on the characteristic features of that item. This task in any classification system is performed by a classifier that takes a feature vector as an input and responds with a category to which the object belongs. A feature vector is a set of features extracted from the input data. To be able to extract features, the unwanted data from the image has to be removed. This is where segmentation algorithms are applied. In this study we make use of three segmentation approaches such as level sets, fuzzy c-means and segmentation based on textural information.

Level sets were first described in 1988 by Osher and Sethian ${ }^{18}$ as a method for capturing moving fronts. In the level set formulation, the segmentation problem is equivalent to the computation of a surface $\Gamma(t)$ that propagates 
in time along its normal direction. The $\Gamma$ surface is also called a propagating front, which according to Osher and Sethian ${ }^{18}$ is embedded as a zero level of a time-varying higher dimensional function $\phi(x, t)$ :

$$
\Gamma(t)=\left\{x \in \mathcal{R}^{3} / \phi(x, t)=0\right\}
$$

An evolution equation for $\phi$, from which we can determine $\Gamma$, where $\Gamma$ is a closed curve in $R^{2}$, can be written in a general form as: ${ }^{19}$

$$
\frac{\partial \phi}{\partial t}+F|\nabla \phi|=0
$$

The function $\phi$ describes a curve defined by $\phi(x, t)=d$, where $d$ is a signed distance between $x$ and the surface $\Gamma$. If $x$ is inside (outside) of $\Gamma$ then $d$ is negative (positive). Function $F$ is a scalar speed function that depends on image data and the function $\phi$.

The main drawback of this procedure is that during the evolution, $\phi$ can assume sharp or flat shapes. To overcome this problem $\phi$ is initialized as a signed distance function before evolution. Later, during evolution, it is periodically reshaped to be a signed distance function. ${ }^{11}$

Here, the approach proposed by $\mathrm{Li}$ et al. ${ }^{11}$ is used. The authors proposed a modification of the traditional variational level sets of Osher and Sethian ${ }^{18}$ to overcome the problem of reshaping function $\phi$ to be a distance function within the evolution cycle. They proposed an evolution equation of the form:

$$
\frac{\partial \phi}{\partial t}=-\frac{\partial \mathcal{E}}{\partial \phi}
$$

where $\frac{\partial \mathcal{E}}{\partial \phi}$ is a Gateaux derivative of the energy function $\mathcal{E}$ and is represented by:

$$
\frac{\partial \mathcal{E}}{\partial \phi}=-\mu\left[\Delta \phi-\operatorname{div}\left(\frac{\nabla \phi}{|\nabla \phi|}\right)\right]-\lambda \delta(\phi) \operatorname{div}\left(g \frac{\nabla \phi}{|\nabla \phi|}\right)-\nu g \delta(\phi),
$$

where $\Delta$ is the Laplacian operator, div is the divergence operator, $\mu>0$ is a parameter controlling the effect of penalizing the deviation of $\phi$ from a signed distance function, $g$ is an edge indicator function, $\lambda>0$ and $\nu$ are constants.

Another segmentation method taken into consideration is a fuzzy approach of Klir and Yuan ${ }^{20}$ that can be used to partition the image information to extract nuclei. In general, a set of data $X=\left\{x_{1}, x_{2}, \ldots, x_{n}\right\}$ is supposed to be divided into $c$ clusters with assumption that $P=\left\{A_{1}, A_{2}, \ldots, A_{c}\right\}$ is known pseudo-partition where $A_{i}$ is a vector of all memberships of $x_{k}$ to cluster $i$. Now, the centers of the $c$ clusters can be calculated by the following equation: ${ }^{21}$

$$
v_{i}=\frac{\sum_{k=1}^{n}\left[A_{i}\left(x_{k}\right)\right]^{m} x_{k}}{\sum_{k=1}^{n}\left[A_{i}\left(x_{k}\right)\right]^{m}}, \quad i=1,2, \ldots, c
$$

where $m>1$ is a weight that controls the fuzzy membership. The memberships are defined by equation 6 below if $\left\|x_{k}-v_{i}\right\|^{2}>0$ for all $i \in\{1,2, \ldots, c\}$. If $\left\|x_{k}-v_{i}\right\|^{2}=0$ for some $i \in I \subseteq\{1,2, \ldots, c\}$ the memberships are defined as a nonnegative real number satisfying equation 7 below for $i \in I$.

$$
\begin{gathered}
A_{i}\left(x_{k}\right)=\left[\sum_{j=1}^{c}\left(\frac{\left\|x_{k}-v_{i}\right\|^{2}}{\left\|x_{k}-v_{j}\right\|^{2}}\right)^{\frac{1}{m-1}}\right]^{-1} \\
\sum_{i \in I} A_{i}\left(x_{k}\right)=1
\end{gathered}
$$

The clustering algorithm seeks a set $P$ that minimizes the performance index $J_{m}(P)$ which is defined by the following:

$$
J_{m}(P)=\sum_{k=1}^{n} \sum_{i=1}^{c}\left[A_{i}\left(x_{k}\right)\right]^{m}\left\|x_{k}-v_{i}\right\|^{2} .
$$

The optimal solution to this problem was presented by Bezdek. ${ }^{22}$ The third segmentation technique we used was image textural description based on second order statistics to generate grey level co-occurrence texture 
features. ${ }^{23}$ Here, for a spatial window inside the image the conditional joint probabilities, $C_{i j}$ are calculated according to the following for all pairwise combinations of grey levels assuming that distances between pixels are known:

$$
C_{i j}=\frac{P_{i j}}{\sum_{i, j=0}^{G-1} P_{i j}},
$$

where $P_{i j}$ is a frequency of occurrence of two grey levels $i$ and $j$ and $G$ is a number of quantized grey levels. The probabilities are stored in a gray level co-occurrence matrix, where $(i, j)$ element of the matrix represents the probability $C_{i j}$. To identify textures within an image we derive four features from the dependency matrix. The four features consist of entropy, correlation, inertia (correlation) and energy. These properties are described by the following equations:

$$
\begin{gathered}
\text { Entropy }=-\sum_{i, j=0}^{G-1} C_{i j} \ln C_{i j}, \\
\text { Contrast }=\sum_{i, j=0}^{G-1} C_{i j}(i-j)^{2}, \\
\text { Inertia }=\sum_{i, j=0}^{G-1} \frac{\left(i-\mu_{x}\right)\left(j-\mu_{y}\right) C_{i j}}{\sigma_{x} \sigma_{y}}, \\
\text { Energy }=\sum_{i, j=0}^{G-1} C_{i j}^{2},
\end{gathered}
$$

where $\sigma$ is a standard deviation and $\mu$ is a mean.

From all compared segmentation techniques fuzzy c-means algorithm doesn't require any additional processing and was applied to segment color information in the image. The remaining two techniques require the image to be converted to gray scale. Textural segmentation uses a typical average conversion to gray scale, where a gray level value is an average of the RGB channels. Level set segmentation requires an initial contour, called initial level set, which was obtained by thresholding of the image red channel which provides best information about nuclear structures out of the three RGB channels. During the staining process nuclei stain with shades purple and when red channel is extracted all the nuclear features are preserved while the background information is lost.

Based on the segmentation results five features were extracted: area, perimeter, eccentricity, convexity and texture measure. Area is calculated as the sum of all pixels of the segmented nucleus. Perimeter is the length of the nuclear envelope, calculated as length of the polygonal approximation of the boundary. Convexity is calculated as the ratio of nucleus area and its convex hull, ${ }^{24}$ which is the minimal area of the convex polygon that can contain the nucleus. Eccentricity is calculated as the ratio of the distance between the foci of an ellipse, that has the same second-moments as the extracted nuclei, and its major axis length.

Classifiers used in this study include Multilayer Perceptron (MLP), Self-Organizing Maps (SOM), Principal Component based Neural Network (PCA) and Support Vector Machines (SVM). MLP is a collection of neurons (a device with many inputs and one output that are trained to fire, or not, for particular input patterns) that are connected to one another. Each connection is assigned an initial weight during the training process. These weights are then adjusted to give a proper answer. The final decision is based on the interaction of weights and the feature vector. MLP is trained using backpropagation learning algorithm in a supervised manner. ${ }^{25}$ A backpropagation algorithm adjusts weights of the network by propagating the output layer values of the network back towards the input layer through all hidden layers. SOMs networks reduce the input space into representative features with the use of self-organizing neural networks. ${ }^{26}$ SOMs are trained in an unsupervised manner to produce a map of similarities of the input data by grouping the similar data items together. PCA are a combination of supervised and unsupervised trained neural networks. At first principal components are found in unsupervised manner from input data and then the supervised MLP is used for classification of the components. ${ }^{27}$ In SVM the classification process is based on nonlinear transformation of a feature vector into a higher dimensional space where a separating hyperplane is constructed. During the training process only those 
vectors that are closest to the separating plane are used because they carry the most valuable information about classification. ${ }^{28}$

\section{RESULTS}

This section presents some of the obtained nuclear segmentation results and demonstrates the performance of the tested classifiers. In the experiments 110 images of fine needle aspirates were used with known malignancy grades collected at the Department of Pathology of Medical University of Wrocław, Poland. All of the images in the database were stained with the Haematoxylin and Eosin technique (HE) which yielded purple and black stain for nuclei, shades of pink for cytoplasm and orange/red for red blood cells. All the images were obtained by Olympus BX 50 microscope with mounted CCD-IRIS camera connected to a PC computer with MultiScan Base 08.98 software.

There were 44 images with high malignancy (G3) and 66 images with intermediate malignancy (G2). The benchmark grades were assigned by an expert pathologist. Since 2005 none of the performed biopsies were assigned with a low malignancy grade, therefore all cases included in the database are graded as intermediate or high malignancy. Here, each classifier was trained with $30 \%$ of all intermediate malignancy cases and $50 \%$ of high malignancy cases. All the remaining cases were used to create a testing set. Examples of the extracted features for each segmentation technique are presented in Table 1.

\begin{tabular}{|c|c|c|c|c|c|c|c|}
\hline Feature & $\begin{array}{l}\text { Segmentation } \\
\text { technique }\end{array}$ & Case 1 & Case 2 & Case 3 & Case 4 & Case 5 & Case 6 \\
\hline \multirow{3}{*}{ Area } & Level set & 417 & 506 & 442 & 3045 & 3802 & 1611 \\
\hline & Fuzzy C & 288 & 381 & 500 & 12786 & 8928 & 11370 \\
\hline & Textural & 100 & 288 & 142 & 303 & 187 & 152 \\
\hline \multirow{3}{*}{ Perimeter } & Level set & 75 & 91 & 82.1 & 265.5 & 280 & 166 \\
\hline & Fuzzy C & 60 & 69 & 77 & 533 & 279 & 353 \\
\hline & Textural & 33 & 60 & 38 & 61 & 48 & 38 \\
\hline \multirow{3}{*}{ Eccentricity } & Level set & 0.6519 & 0.7517 & 0.7012 & 0.6820 & 0.8000 & 0.7000 \\
\hline & Fuzzy C & 0.6125 & 0.6435 & 0.6345 & 0.6412 & 0.7342 & 0.7206 \\
\hline & Textural & 0.5697 & 0.6538 & 0.6701 & 0.6698 & 0.5410 & 0.6002 \\
\hline \multirow{3}{*}{ Convexity } & Level set & 0.9309 & 0.9038 & 0.9085 & 0.8580 & 0.8000 & 0.9000 \\
\hline & Fuzzy C & 0.9422 & 0.9533 & 0.9491 & 0.9007 & 0.9561 & 0.9255 \\
\hline & Textural & 0.9539 & 0.9396 & 0.9743 & 0.9272 & 0.9330 & 0.9677 \\
\hline \multirow{3}{*}{ Texture } & Level set & 122.4 & 124 & 104.6 & 91.4 & 60.5 & 59.1 \\
\hline & Fuzzy C & 115.8 & 116.9 & 108.9 & 105 & 71 & 84 \\
\hline & Textural & 80.3 & 86.8 & 31 & 32.8 & 14.4 & 5.2 \\
\hline \multicolumn{2}{|c|}{ BR Grade } & G3 & G3 & G3 & G2 & G2 & G2 \\
\hline
\end{tabular}

Table 1. Some of the calculated features along with pathologist grading

From the table we can see the influence of the nuclei representation obtained with all three segmentations on class separation. Fig. 2 shows the obtained nuclear representations after application of the three compared approaches. Fig. 3 shows the typical nuclear boundaries calculated during the segmentation process and compares level set, fuzzy c-means and textural segmentation approaches.

a)

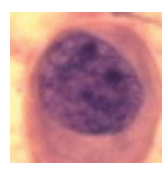

b)

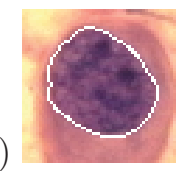

c)

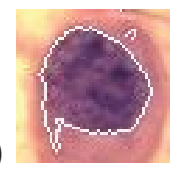

d)

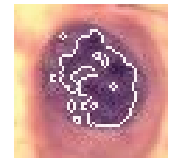

Figure 2. Nuclear boundaries: a) Original, b) obtained with level sets, c) obtained with fuzzy c-means, d) obtained with textural segmentation.

The presented figures support our findings on influence of segmentation on the feature extraction and further 

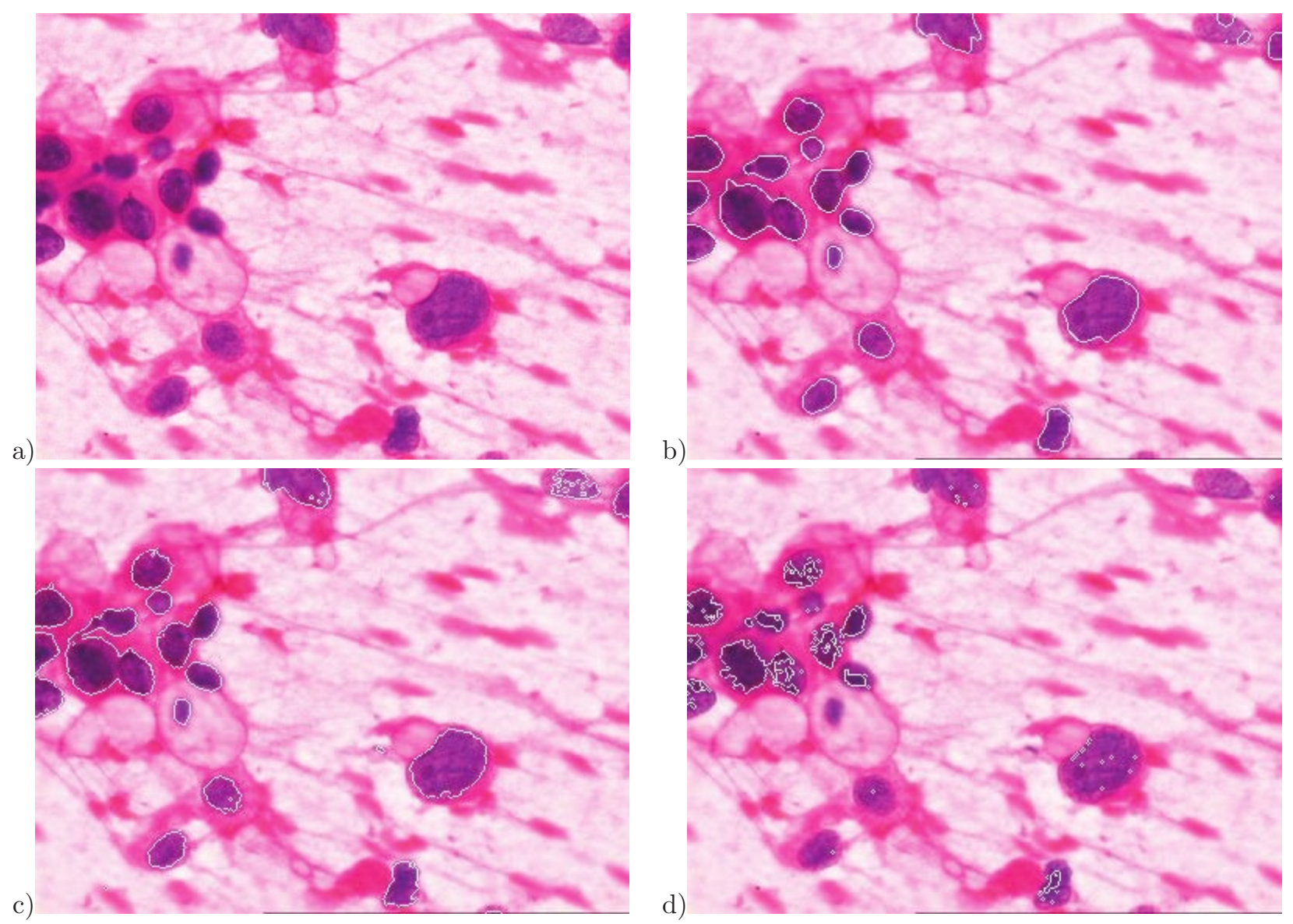

Figure 3. Segmentation results: a) Original, b) obtained with level sets, c) obtained with fuzzy c-means, d) obtained with textural segmentation.

\begin{tabular}{|c|c|c|c|}
\hline \multirow{2}{*}{ Classifier } & \multicolumn{3}{|c|}{ Segmentation technique } \\
\cline { 2 - 4 } & Level set & Fuzzy c-means & Textural \\
\hline SOM & $6.32 \%$ & $15.66 \%$ & $25.67 \%$ \\
MLP & $6.73 \%$ & $3.07 \%$ & $23 \%$ \\
SVM & $5.76 \%$ & $22 \%$ & $22.58 \%$ \\
PCA & $7.22 \%$ & $16.72 \%$ & $25.19 \%$ \\
\hline Avg. & $6.51 \%$ & $14.36 \%$ & $24.11 \%$ \\
\hline \multicolumn{4}{|c}{ Table 2. Recorded error rates } \\
\hline
\end{tabular}

on classification. All classifiers were trained and tested with the previously described features. Classification performance of all classifiers is summarized in Table 2 and obtained error rates for all tests show that MLP performed best for features extracted from images segmented with fuzzy c-means algorithm while all the remaining classifiers achieved best performance for images segmented with level sets. This is also reflected by calculated average performance of each segmentation technique. 


\section{CONCLUSIONS}

In this work, the influence of different types of nuclear segmentation from fine needle aspiration biopsy images on breast cancer malignancy classification is presented. Section 2 describes used segmentation techniques and tested classifiers. The presented results show the behavior of the three segmentation algorithms from which the following conclusions can be drawn:

1. Level sets segmentation

- Precise method for boundary representation.

- This method requires an initial level set - initial boundary.

- The running time of level sets is much faster than the running time of another segmentation technique called the Hough transform, see Jelen et al. ${ }^{29}$ The initial boundary can be obtained with thresholding which reduces the computational load.

- Provides a very good trade-off between the running time and boundary representation precision.

2. Fuzzy c-means segmentation

- This method is based on color classification of the neighboring pixels.

- Provides good representation of the boundaries.

- The boundary representation is better than that obtained with Hough transform and textural segmentation but not as good as with level sets.

- Fuzzy c-means does not require any initial boundary.

3. Textural segmentation

- Segmentation is based on textural description of the nuclei.

- Similarly to fuzzy c-means, gray level quantization does not require any initial contours.

- The boundary representation is better than with Hough transform but not as precise as with either level sets or fuzzy c-means.

In general, it can be noticed that although fuzzy c-means provides better nuclei segmentation then textural segmentation, the level sets represent the nuclear boundary the most precisely. It can easily be noticed in Fig. 3 that textural segmentation algorithm loses a lot of nuclear information during the segmentation.

The results presented in section 3 support our findings that level set segmentation provides most valuable nuclear information when compared with the remaining two approaches. This conclusion is also supported by the achieved classification rates presented in Table 2. Here we can notice that MLP classifier performs best for features extracted from nuclei obtained by fuzzy c-means segmentation yielding an error rate of $3.07 \%$. It can also be seen that textural segmentation provides the worst representation of nuclei, which is supported by the error rates achieved for all tested classifiers. We can conclude that, on average, level sets are a better choice for segmentation achieving $6.51 \%$ average error rate. Finally, we can conclude that the representation of nuclei obtained by segmentation strongly influences the classification performance.

\section{REFERENCES}

[1] Bloom, H. and Richardson, W., "Histological grading and prognosis in breast bancer," British Journal of Cancer 11, 359-377.

[2] Cross, S., "Grading and scoring in histopathology," Histopathology 33(2), 99-106 (1998).

[3] Street, N., "Xcyt: a system for remote cytological diagnosis and prognosis of breast cancer," in [Soft Computing Techniques in Breast Cancer Prognosis and Diagnosis], Jain, L., ed., 297-322, World Scientific Publishing, Singapore (2000).

[4] Walker, H. J. and Albertelli, L., "Breast cancer screening using evolved neural networks," IEEE International Conference on Systems, Man, and Cybernetics, San Diego, USA 2, 1619-1624 (1998). 
[5] Cheng, H., Li, X., Riodan, D., and J.N., S., "A parallel approach to tubule grading in breast cancer lesions and its VLSI implementation," Computer-Based Medical Systems: Fourth Annual IEEE Symposium, 322329 (1991).

[6] Schnorrenberg, F., Tsapatsoulis, N., Pattichis, C., Schizas, C., Kollias, S., Vassiliou, M., Adamou, A., and Kyriacou, K., "A modular neural network system for the analysis of nuclei in histopathological sections," IEEE Engineering in Medicine and Biology Magazine 19, 48-63 (2000).

[7] Jeleń, Ł., Fevens, T., and Krzyżak, A., "Classification of breast cancer malignancy using cytological images of fine needle aspiration biopsies," Int. J. Math. Comput. Sci. 18(1), 75-83 (2008).

[8] Cheng, H., Wu, C., and Hung, D., "Vlsi for moment computation and its application to breast cancer detection," Pattern Recognition 31(8), 1391-1406 (1998).

[9] Street, W. N., Wolberg, W. H., and Mangasarian, O. L., "Nuclear feature extraction for breast tumor diagnosis," in [Imaging Science and Technology/Society of Photographic Instrumentation Engineers 1993 International Symposium on Electronic Imaging: Science and Technology, ISET/SPIE], 1905, 861-870 (1993).

[10] Nezafat, R., Tabesh, A., Akhavan, S., Lucas, C., and Zia, M., "Feature selection and classification for diagnosing breast cancer," Proceedings of International Association of Science and Technology for Development International Conference, IASTED, Cancun, Mexico, 310-313 (1998).

[11] Li, C., Xu, C., Gui, C., and Fox, M., "Level set evolution without re-initialization: a new variational formulation.," Proceedings of the IEEE Conference on Computer Vision and Pattern Recognition, CVPR 2005, San Diego, USA , 430-436 (2005).

[12] Tsai, A., Yezzi, A., Wells, III, W., Tempany, C., Tucker, D., Fan, A., Grimson, W., and Willsky, A., "A shape-based approach to the segmentation of medical imagery using level sets," IEEE Transactions on Medical Imaging 22, 137-154 (February 2003).

[13] Ardizzone, E., Pirrone, R., and Gambino, O., "Fuzzy c-means segmentation on brain mr slices by rfinhomogeneity," in [Applications of Fuzzy Sets Theory], 4578, 378-384 (2007).

[14] Schmid, P. and Fischer, S., "Colour segmentation for the analysis of pigment skin lesions," in [Proceedings of the Sixth International Conference on Image Processing and Applications], 2, 688-692 (1997).

[15] Umbaugh, S., Wei, Y., and Zuke, M., "Feature extraction in image analysis," IEEE Engineering in Medicine and Biology Magazine 16(4), 62-73 (1997).

[16] Sabino, D., da Fontoura Costa, L., Rizzatti, E., and Zago, M., "A texture approach to leukocyte recognition," Real-Time Imaging 10, 205-216 (2004).

[17] Abella, M., Zubeldia, J., Conejero, L., Malpica, N., A.Santos-Lleo, and Desco, M., "Preliminary results on automatic quantification of histological studies in allergic asthma," in [Proceedings of 1st Workshop on Microscopic Image Analysis with Applications in Biology (in conjunction with MICCAI, Copenhagen)], 50-54 (2006).

[18] Osher, S. and Sethian, J., "Fronts propagating with curvature-dependent speed: algorithms based on Hamilton-Jacobi formulations.," Journal of Computational Physics 79, 12-49 (1988).

[19] Sethian, J. and Adalsteinsson, D., "An overview of level set methods for etching, deposition, and lithography development," IEEE Transactions on Semiconductor Manufacturing 10(1), 167-184 (1997).

[20] Klir, G. and Yuan, B., [Fuzzy Sets and Fuzzy Logic: Theory and Applications], Prentice Hall, New Jersey (1995).

[21] Theera-Umpon, N., "Patch-Based white blood cell nucleus segmentation using fuzzy clustering," ECTI Transactions on Electrical Engineering, Electronics and Communications 3(1), 15-19 (2005).

[22] Bezdek, J., [Pattern Recognition with Fuzzy Objective Function Algorithms], Plenum Press, New York (1981).

[23] Haralick, R., Shanmugam, K., and Dinstein, I., "Textural features for image classification," IEEE Transactions on Systems, Man, and Cybernetics 3(6), 610-621 (1973).

[24] Zunic, J. and Rosin, P., "A convexity measurement for polygons.," British Machine Vision Conference, Cardiff, UK 24, 173-182 (2002).

[25] Duda, R., Hart, P., and Stork, D., [Pattern Classification], Wiley Interscience Publishers, New York, 2nd ed. (2001). 
[26] Kohonen, T., "The self-organizing map," in [Proceedings of Institute of Electrical and Electronics Engineers], 78, 1464-1480 (1990).

[27] Oja, E., "A simplified neuron modeled as a principal component analyzer," Journal of Mathematical Biology 15, 267-273 (1982).

[28] Friess, T., Cristianini, N., and Campbell, C., "The kernel adatron algorithm: a fast and simple learning procedure for support vector machines," in [15th International Conference on Machine Learning, ICML-98, Morgan Kaufman Publishers, Madison, WI, USA], 188-196 (1998).

[29] Jeleń, Ł., Krzyżak, A., and Fevens, T., "Automated feature extraction for breast cancer grading with Bloom-Richardson scheme," International Journal of Computer Assisted Radiology and Surgery 1(1), 468469 (2006). 